\section{Vestibulocochlear Nerve}

John E. Mendoza

Department of Psychiatry and Neuroscience, Tulane Medical School and SE Louisiana

Veterans Healthcare System, New Orleans, LA, USA

\section{Synonyms}

Eighth cranial nerve

\section{Definition}

The cranial nerve that subserves both hearing and vestibular functions. It is essentially a double nerve that enters the brainstem laterally at the pontomedullary junction.

\section{Current Knowledge}

The cochlear portion of this nerve is responsible for carrying auditory information from the hair cells of the organ of Corti in the cochlea, within the inner ear, to the dorsal and ventral cochlear nuclei in the medulla. The vestibular portion of the nerve is derived from nerve receptors in the three semicircular canals, the utricle, and the saccule. The semicircular canals represent three different planes or orientations in space and respond to angular acceleration and deceleration. The utricle responds to gravitational forces and horizontal linear acceleration. The saccule responds to linear acceleration in the dorsal-ventral plane. Together these responds provide information regarding the orientation of the head in space and the movement of one's body (head) through space (both the direction of movement, as well as the sense of movement). As the fibers for the vestibular system enter the brainstem, a few course directly to the flocculonodular lobe of the cerebellum, while most synapse in the vestibular nuclei of the medulla and pons.

The vestibulocochlear nerve can be affected by various neuropathological processes, including tumors, infections, and strokes. If the cochlear portion of the nerve is damaged, reductions in or loss of hearing as well as tinnitus may result. If the vestibular portion of the nerve is affected, the patient may experience vertigo and/or unsteadiness of gait.

\section{Cross-References}

Auditory System

- Vestibular System

\section{Reference and Readings}

Wilson-Pauwek, L., Akesson, E. J., Stewart, P. A., \& Spacey, S. D. (2002). Cranial nerves in health and disease. Hamilton: B.C. Decker. 